Bangladesh J. Bot. 48(4): 1047-1063, 2019 (December)

\title{
EVALUATION OF SALINE-ALKALI AND DROUGHT TOLERANCE IN MAIZE VARIETIES
}

\author{
Huili Zhang, Chuang Yuan, Guilian Mao ${ }^{1}, \mathrm{Xue} \mathrm{GaO}^{2,3}$, \\ LIN Z $\mathrm{HU}^{2,3}$ AND XING XU ${ }^{2,3 *}$ \\ Agriculture College of Ningxia University, Yinchuan 750021, China
}

Keywords: Maize, Saline-alkali stress, Drought stress, Principle component analysis, Subordinate function method, Cluster analysis

\begin{abstract}
Saline-alkali and drought stresses are one of the abiotic stress factors that limit the normal growth and development of plants. In this work, various agronomic indexes including growth physiology and yield attributes were studied under saline-alkali and drought stress treatments. It was found that the limit of plant growth and development caused by drought stress is much higher than that of saline-alkali stress $(\mathrm{p}<0.01)$. Based on the comprehensive evaluation value ( $\mathrm{D}$ value), under saline-alkali stress condition, 36 maize varieties could be divided into four groups by cluster analysis (CA): High saline-alkali tolerance ( 3 varieties), medium saline-alkali tolerant(10 varieties), saline-alkali sensitive (19 varieties), high saline-alkali sensitive (4 varieties). In drought stress condition, 36 maize varieties could be divided into five groups by cluster analysis (CA): High drought-tolerance (2 varieties), medium drought-tolerant (14 varieties), low drought-tolerant (15 varieties), drought-sensitive (4 varieties), high drought-sensitive (1 variety). Therefore, this study provides a comprehensive screening of maize varieties under saline-alkali and drought stresses.
\end{abstract}

\section{Introduction}

The Northwestern China is located in arid and semi-arid regions, with less rainfall and extremely high evaporation. Plants suffer due to drought and salinity (Glemm et al. 1999, Zhang et al. 2017).

Many workers studied morphological structures (Rodrí Guezet al. 2005, Kutlu et al. 2009, Zhang et al. 2015), physiology and biochemistry (Guan et al. 2015), molecular level (Trachsel et al. 2010) of plants under saline-alkali or drought stresses in laboratory and greenhouse conditions (Zhang et al. 2011, Hao et al. 2013, Du et al. 2015), when information is scanty under field condition in general maize (Zea mays L.) in paticular. Maize as an important staple food, fodder crop and industrial raw material are widely grown in China, but it is moderately sensitive to saline-alkali and drought stress (Fu et al. 2009) thus yield is reduced severely. So, it is imperative to optimize maize yield under drought and saline-alkali stresses. The low-cost management is screening maize varieties (lines) as induced by the stress variabilities (Lu and Wang 2004, Takehisa et al. 2004, Akram et al. 2010). Hence, this study was made with 36 varieties of maize to select tolerance and sensitive lines for distribution in dry and salinized land.

\section{Materials and Methods}

The study site included Pingluo (latitude $38^{\circ} 53^{\prime} \mathrm{N}$, longitude $106^{\circ} 33^{\prime} \mathrm{E}$, altitude $1098.7 \mathrm{~m}$, annual temperature $9.4^{\circ} \mathrm{C}$, annual precipitation $177.8 \mathrm{~mm}$ ) and Tongxin (latitude $36^{\circ} 58^{\prime} \mathrm{N}$, longitude $105^{\circ} 54^{\prime} \mathrm{E}$, altitude $1339.3 \mathrm{~m}$, annual temperature $9.5^{\circ} \mathrm{C}$, annual precipitation 259.8 $\mathrm{mm})$, Ningxia Hui Autonomous Region, China.

*Author for correspondence: <xuxingscience@126.com>. ${ }^{1}$ Life Science College of Ningxia University, Yinchuan-750021, China. ${ }^{2}$ Breeding Base of State Key Laboratory of Land Degradation and Ecological Restoration of North-western China, Ningxia University, Yinchuan-750021, China. ${ }^{3}$ Key Laboratory for Restoration and Reconstruction of Degraded Ecosystem in North-western China, Ministry of Education, Ningxia University, Yinchuan-750021, China. 
Field experiment was carried out in randomized complete block design with three replications, maintaining row spacing of $60 \mathrm{~cm}$, plant spacing of $20 \mathrm{~cm}$. Thirty-six maize varieties were sown on 26th of April. Irrigation was carried out only for ensuring seedling emergence in drought tolerance test. The rainfall during the whole growth period was $146.80 \mathrm{~mm}$. The saline-alkali tolerance test was carried out under conditions of $5.656 \mathrm{~g} / \mathrm{kg}$ total soluble salinity content and $\mathrm{pH}$ 8.71. Fertilizer was applied as $\mathrm{N}: \mathrm{P}_{2} \mathrm{O}_{5}: \mathrm{K}_{2} \mathrm{O}=15: 15: 15$. Intercultural operations were done as and when required.

The tested varieties were H242/1522, A49/1522, A49/1528, A3Gao/1519, , H237/A26, A18/1506, 133-2/1528, H242/1523, A3/H237, 1421/1522, H237/1519, Bei21/1520, Ning3-1/1528, H14/1528, Bei21/1522, Ning3-2/1528, A3Gao/A18, H237/A3, H237/A18, 1517/Xun11, Xun11/1517, H14/A18, Gui Silage Numeber 1, Ke Duo 8, TY1, H398/1523, C5/1521, A28/1522, A133/1528, A133/1506, 5178-1/1528, A58-1/1501, A28/1523, 1522/H237, Qing Feng 5 and Zhong Yuan Dan32 and they are numbered from 1 to 36 in order.

Relative chlorophyll content of leaves (Chl) was monitored by using a portable SPAD-502 meter (Minolta, Osaka, Japan). The degree of chlorosis was estimated non-destructively in the youngest fully expanded apical leaves from each treatment. Six SPAD readings were recorded for each leaf, in a relative position of $2 / 3$ of the distance relative to leaf base. Measurements of photosynthetic rate, stomatal conductance and transpiration rate were made on a fully expanded leaf of each plant by using LI-6400 series portable photosynthesis system (LI-COR Company of USA). These measurements were made at sunny day (from 9.30 to 11.30 a.m.) at maturity.

The hand-held canopy temperature detector was used, to measure the CT value of each varieties. Ten plants and five ears were selected randomly from each plot for determining growth, yield attributes and yield.

Microsoft Excel 2007 was used for data collection, DPS 7.05 software was used for significance and correlation analysis, and then the SPSS 20.0 statistical software was used for principal component analysis (PCA) (Karamizadeh et al. 2013) and cluster analysis. The results are expressed as mean values \pm SE (Standard error).

The method of membership function was used to comprehensively evaluate the drought tolerance and saline-alkali tolerance of each maize varieties during mature period (Chen 2002, Shi et al. 2010, Bo et al. 2017).

Firstly, the formula $U\left(X_{j}\right)=\left(X_{j}-X_{\min }\right) /\left(X_{\max }-X_{\min }\right)(j=1,2,3, \ldots, n)$ was used, where $X_{j}$ is the measured value of a certain indicator. And $X_{\min }, X_{\max }$ are expressed as the minimum and maximum values of a certain index of 36 tested maize varieties, respectively.

Secondly, according to the relative importance of the traits, a certain weight is assigned. Weight: $\mathrm{W}_{\mathrm{j}}=\mathrm{P}_{\mathrm{j}} / \Sigma_{\mathrm{j}=1}^{\mathrm{n}} \mathrm{Pj}(\mathrm{j}=1,2,3, \ldots, \mathrm{n})$, where $\mathrm{W}_{\mathrm{j}}$ denotes the major degree of the jth factor in all common factors. $P_{j}$ is the correlation coefficient between the jth index and the drought tolerant coefficient or saline-alkali tolerance coefficient of each material and represents the contribution rate of the jth common factor of each varieties.

Finally, the value of the membership function of each variety was added, and takes it as the comprehensive evaluation value (D value). $\left.D=\sum_{[=1}[U \times]\right) \times W_{i]}(j=1,2,3, \ldots, n)$, where $D$ is the integrated evaluation value of tested material under saline-alkali and drought stress conditions. According to the size of D value, the strength of saline-alkali tolerance and drought tolerance of maize varieties be determined. The greater of the $\mathrm{D}$ value, the varieties drought tolerance and saline-alkali tolerance would be stronger, on the contrary, drought and saline-alkali would be weaker. 


\section{Results and Discussion}

Results revealed that the growth and yield attributions of different varieties/lines vary significantly under different stress conditions (Tables 1 and 2). Plant height varied from 235.33 to $396.78 \mathrm{~cm}$ and 142.00 to $267.89 \mathrm{~cm}$ due to saline-alkali and drought stresses, respectively. Under saline-alkali stress treatment, the plant height of three lines (133-2/1528, H242/1523 and Ning3-1/ 1528) was high, and the plant height of Zhong Yuan Dan 32 was the lowest. Under drought stress treatment, the plant height of varieties No.28, 29 and 33 was significantly higher, and Gui Silage Numeber 1 and Zhong Yuan Dan 32 was the lowest. The stem diameter ranged as $21.93-32.76$ $\mathrm{mm}$ and $15.67-28.82 \mathrm{~mm}$ under saline-alkali and drought treatments, respectively when the variety No.6 showed maximum stem diameter in saline-alkali and drought condition. On the other hand, variety No. 36 showed minimum stem diameter under saline-alkali and drought conditions. SPAD value varies from 24.58 to 51.62 and 21.37 to 54.73 under saline-alkali and drought stresses, respectively. The line of $\mathrm{H} 242 / 1522$ was the highest, the variety of A18/1506 and H14/1528 were the lowest under saline-alkali condition. Under dry cultivation condition, the A133/1506 showed maximum SPAD value, Zhong Yuan Dan 32 showed minimum SPAD value. Under different stresses conditions, the canopy temperature of different varieties was different. Zhong Yuan Dan 32 showed the highest under drought and saline-alkali stresses. The above results showed that there is a clear difference between the same indicators of different varieties.

The results in Table 2 showed that under different stress conditions, the photosynthetic parameters and stay-green ability of different varieties had different changes. For the net photosynthetic rate $\left(P_{n}\right)$, both stress conditions showed the highest was $133-2 / 1528$, the lowest net photosynthetic rate $\left(\mathrm{P}_{\mathrm{n}}\right)$ was A58 - 1/1501 under saline-alkali stress, and the lowest was Zhong Yuan Dan 32 under dry land. Stomatal conductance $\left(\mathrm{G}_{\mathrm{s}}\right)$ and transpiration rate $\left(\mathrm{T}_{\mathrm{r}}\right)$ of Zhong Yuan Dan 32 were highest in saline-alkali condition, and the stomatal conductance $\left(G_{s}\right)$ and transpiration rate $\left(\mathrm{T}_{\mathrm{r}}\right)$ of Zhong Yuan Dan 32 were significantly higher than those of other varieties $(\mathrm{p}<0.05)$. Stomatal conductance $\left(\mathrm{G}_{\mathrm{s}}\right)$ of $5178-1 / 1528$ was the highest under dry land cultivation condition, stomatal conductance $\left(\mathrm{G}_{\mathrm{s}}\right)$ and transpiration rate $\left(\mathrm{T}_{\mathrm{r}}\right)$ of Zhong Yuan Dan 32 was the lowest. The above indices can reflect the salt and drought tolerance of the varieties from different angles. The evaluation standard, index system about the salt and drought tolerance of plants is different. A single index cannot accurately reflect the salt and drought tolerance of each varieties.

In this experiment, the correlations between agronomy indices, photosynthetic parameters and yield indices of 36 different maize varieties under saline-alkali stress were analyzed. It has been shown in Fig. 1 that there were some correlations between the related indicators at mature stage. Significant positive correlations were observed between plant height and stem diameter, stay-green ability, above ground biomass, SPAD values and stay-green ability, stay-green ability and net photosynthetic rate, above ground biomass, stomatal conductance and transpiration rate, dry fresh ratio and stomatal conductance, transpiration rate, 100-grain weight, single ear weight and 100-grain weight, ear length, row grains, bald length and ear length. Significant negative correlations were observed between canopy temperature and plant height, above ground biomass, dry fresh ratio and stay-green ability, net photosynthetic rate, bald length and stay-green ability, net photosynthetic rate, row grains.

The results demonstrated that the correlation analysis about various indicators of maize varieties at mature stage under drought stress, the data can be seen from the Fig. 2. Significant positive correlations were observed between plant height and SPAD value, stay-green ability, aboveground biomass, SPAD value and stay-green ability and aboveground biomass, stomatal conductance and transpiration rate, single ear weight and 100-grain weight, ear diameter, ear length and ear diameter, axis diameter, ear row number, ear diameter and axis diameter, ear row 
Table 1. Effects of saline-alkali and drought stresses on plant height $(\mathrm{cm})$, stem diameter (mm), SPAD values and canopy temperature of maize varieties.

\begin{tabular}{|c|c|c|c|c|}
\hline \multirow[t]{2}{*}{ No. } & Saline alkali & Drought land & Saline alkali & Drought land \\
\hline & Plant height $(\mathrm{cm})$ & Plant height $(\mathrm{cm})$ & Stem diameter $(\mathrm{mm})$ & Stem diameter $(\mathrm{mm})$ \\
\hline 1 & $357.22 \pm 15.60 \mathrm{a}-\mathrm{e}$ & $192.67 \pm 4.73 \mathrm{ij}$ & $29.03 \pm 2.30 \mathrm{a}-\mathrm{e}$ & $24.37 \pm 0.33 \mathrm{~d}-\mathrm{m}$ \\
\hline 2 & $382.67 \pm 4.22 \mathrm{ab}$ & $197.78 \pm 5.35 \mathrm{hi}$ & $29.57 \pm 0.59 a-e$ & $22.76 \pm 1.90 h-q$ \\
\hline 3 & $359.67 \pm 25.70 a-e$ & $172.00 \pm 2.52 \mathrm{~m}-\mathrm{p}$ & $28.10 \pm 0.71 \mathrm{a}-\mathrm{g}$ & $22.47 \pm 0.84 i-q$ \\
\hline 4 & $354.22 \pm 12.13 \mathrm{a}-\mathrm{f}$ & $188.67 \pm 3.93 \mathrm{i}-1$ & $29.19 \pm 2.11 \mathrm{a}-\mathrm{e}$ & $25.07 \pm 0.74 c-j$ \\
\hline 5 & $345.33 \pm 4.00 \mathrm{~b}-\mathrm{h}$ & $220.89 \pm 1.25 \mathrm{de}$ & $24.74 \pm 0.81 \mathrm{~d}-\mathrm{h}$ & $25.63 \pm 0.44 b-h$ \\
\hline 6 & $350.33 \pm 22.00 \mathrm{~b}-\mathrm{h}$ & $223.56 \pm 1.46 \mathrm{de}$ & $32.76 \pm 2.27 \mathrm{a}$ & $28.82 \pm 0.10 \mathrm{a}$ \\
\hline 7 & $396.78 \pm 15.49 a$ & $216.78 \pm 1.44 \mathrm{ef}$ & $28.01 \pm 0.59 \mathrm{a}-\mathrm{g}$ & $25.03 \pm 1.85 \mathrm{c}-\mathrm{j}$ \\
\hline 8 & $396.00 \pm 22.37 \mathrm{a}$ & $215.56 \pm 1.57 \mathrm{e}-\mathrm{g}$ & $30.02 \pm 1.17 \mathrm{a}-\mathrm{e}$ & $27.66 \pm 0.64 a-c$ \\
\hline 9 & $306.78 \pm 12.29 \mathrm{~g}-\mathrm{i}$ & $175.11 \pm 2.38 \mathrm{~m}-\mathrm{o}$ & $26.50 \pm 1.55 b-h$ & $23.92 \pm 0.37 f-n$ \\
\hline 10 & $339.89 \pm 1.75 b-h$ & $190.33 \pm 3.03 \mathrm{i}-\mathrm{k}$ & $29.22 \pm 0.14 a-e$ & $24.21 \pm 0.16 \mathrm{e}-\mathrm{m}$ \\
\hline 11 & $333.00 \pm 9.29 b-h$ & $183.78 \pm 1.06 \mathrm{j}-\mathrm{m}$ & $26.85 \pm 1.33 b-h$ & $26.59 \pm 0.90 \mathrm{a}-\mathrm{f}$ \\
\hline 12 & $311.33 \pm 17.57 \mathrm{f}-\mathrm{i}$ & $204.78 \pm 2.26 \mathrm{gh}$ & $25.94 \pm 1.49 b-h$ & $26.90 \pm 0.07 \mathrm{a}-\mathrm{e}$ \\
\hline 13 & $394.78 \pm 12.52 \mathrm{a}$ & $230.78 \pm 2.26 \mathrm{~cd}$ & $30.04 \pm 2.51 \mathrm{a}-\mathrm{d}$ & $23.83 \pm 0.95 f-n$ \\
\hline 14 & $338.11 \pm 27.90 b-h$ & $217.67 \pm 1.20 \mathrm{ef}$ & $31.05 \pm 1.89 \mathrm{ab}$ & $27.17 \pm 0.77 a-d$ \\
\hline 15 & $367.33 \pm 13.30 a-d$ & $183.44 \pm 2.15 \mathrm{j}-\mathrm{m}$ & $28.38 \pm 0.61 a-f$ & $24.59 \pm 0.43 \mathrm{~d}-1$ \\
\hline 16 & $342.67 \pm 31.35 b-h$ & $208.22 \pm 2.16 f-h$ & $30.50 \pm 2.01 \mathrm{a}-\mathrm{c}$ & $28.18 \pm 1.91 \mathrm{ab}$ \\
\hline 17 & $335.22 \pm 6.78 b-h$ & $180.89 \pm 3.38 \mathrm{j}-\mathrm{m}$ & $28.00 \pm 1.84 \mathrm{a}-\mathrm{g}$ & $23.34 \pm 0.47 \mathrm{~g}-\mathrm{o}$ \\
\hline 18 & $316.78 \pm 3.68 \mathrm{e}-\mathrm{i}$ & $161.11 \pm 1.83 \mathrm{pq}$ & $24.56 \pm 0.68 \mathrm{~d}-\mathrm{h}$ & $22.92 \pm 0.82 \mathrm{~g}-\mathrm{p}$ \\
\hline 19 & $287.45 \pm 13.67 \mathrm{ij}$ & $179.11 \pm 3.16 \mathrm{k}-\mathrm{n}$ & $27.49 \pm 1.14 \mathrm{a}-\mathrm{g}$ & $25.27 \pm 0.75 c-i$ \\
\hline 20 & $369.89 \pm 3.61 \mathrm{a}-\mathrm{c}$ & $167.56 \pm 0.48 n-q$ & $26.77 \pm 1.42 b-h$ & $23.24 \pm 1.20 \mathrm{~g}-\mathrm{o}$ \\
\hline 21 & $356.67 \pm 11.62 a-e$ & $156.44 \pm 4.24 q$ & $28.28 \pm 1.12 \mathrm{a}-\mathrm{g}$ & $23.42 \pm 1.06 \mathrm{~g}-\mathrm{O}$ \\
\hline 22 & $342.22 \pm 9.20 b-h$ & $177.78 \pm 2.281-\mathrm{o}$ & $28.12 \pm 1.26 \mathrm{a}-\mathrm{g}$ & $21.72 \pm 0.721-q$ \\
\hline 23 & $308.11 \pm 9.25 \mathrm{~g}-\mathrm{i}$ & $142.00 \pm 4.60 \mathrm{r}$ & $26.47 \pm 3.09 b-h$ & $24.70 \pm 0.49 \mathrm{~d}-\mathrm{k}$ \\
\hline 24 & $323.22 \pm 3.70 \mathrm{~d}-\mathrm{i}$ & $166.78 \pm 2.61 \mathrm{o}-\mathrm{q}$ & $24.94 \pm 0.81 \mathrm{~d}-\mathrm{h}$ & $19.92 \pm 0.50 q$ \\
\hline 25 & $327.00 \pm 33.41 \mathrm{c}-\mathrm{i}$ & $189.78 \pm 2.72 \mathrm{i}-1$ & $25.27 \pm 0.51 \mathrm{c}-\mathrm{h}$ & $22.27 \pm 0.09 j-q$ \\
\hline 26 & $340.33 \pm 1.45 b-h$ & $235.33 \pm 4.19 \mathrm{c}$ & $27.35 \pm 2.05 \mathrm{~b}-\mathrm{g}$ & $22.82 \pm 0.33 \mathrm{~g}-\mathrm{q}$ \\
\hline 27 & $308.33 \pm 6.33 \mathrm{~g}-\mathrm{i}$ & $249.22 \pm 1.28 b$ & $23.42 \pm 0.60 f-h$ & $21.17 \pm 1.02 \mathrm{n}-\mathrm{q}$ \\
\hline 28 & $339.00 \pm 3.22 \mathrm{~b}-\mathrm{h}$ & $267.89 \pm 2.94 a$ & $25.93 \pm 1.59 b-h$ & $21.61 \pm 1.12 \mathrm{~m}-\mathrm{q}$ \\
\hline 29 & $304.00 \pm 3.06 \mathrm{hi}$ & $263.22 \pm 3.86 a$ & $24.73 \pm 3.10 \mathrm{~d}-\mathrm{h}$ & $22.95 \pm 0.32 g-p$ \\
\hline 30 & $259.00 \pm 7.81 \mathrm{jk}$ & $248.33 \pm 3.47 b$ & $26.71 \pm 0.75 b-h$ & $25.78 \pm 0.19 b-g$ \\
\hline 31 & $361.00 \pm 14.15 \mathrm{a}-\mathrm{e}$ & $258.89 \pm 8.68 \mathrm{ab}$ & $28.68 \pm 1.64 a-f$ & $20.55 \pm 0.72 \mathrm{o}-\mathrm{q}$ \\
\hline 32 & $308.67 \pm 11.26 \mathrm{~g}-\mathrm{i}$ & $235.89 \pm 6.95 \mathrm{c}$ & $29.61 \pm 1.67 \mathrm{a}-\mathrm{e}$ & $20.68 \pm 1.10 \mathrm{o}-\mathrm{q}$ \\
\hline 33 & $351.00 \pm 7.00 \mathrm{~b}-\mathrm{g}$ & $265.11 \pm 8.43 \mathrm{a}$ & $27.27 \pm 1.61 b-h$ & $21.85 \pm 0.18 \mathrm{k}-\mathrm{q}$ \\
\hline 34 & $317.67 \pm 5.24 \mathrm{e}-\mathrm{i}$ & $257.56 \pm 3.40 \mathrm{ab}$ & $22.92 \pm 1.26 \mathrm{gh}$ & $20.64 \pm 1.14 \mathrm{o}-\mathrm{q}$ \\
\hline 35 & $302.00 \pm 5.51 \mathrm{hi}$ & $230.22 \pm 2.12 \mathrm{~cd}$ & $24.54 \pm 2.58 \mathrm{e}-\mathrm{h}$ & $20.24 \pm 0.35 p q$ \\
\hline 36 & $235.33 \pm 8.25 \mathrm{k}$ & $143.00 \pm 5.78 \mathrm{r}$ & $21.93 \pm 0.80 \mathrm{~h}$ & $15.67 \pm 0.44 \mathrm{r}$ \\
\hline Average & 336.20 & 205.50 & 27.30 & 23.56 \\
\hline $\mathrm{CV} / \%$ & 10.49 & 17.53 & 8.82 & 11.41 \\
\hline
\end{tabular}


(Contd.)

\begin{tabular}{|c|c|c|c|c|}
\hline \multirow[t]{2}{*}{ Number } & Saline alkali & Dry land & Saline alkali & Dry land \\
\hline & SPAD values & SPAD values & Canopy temperature $\left({ }^{\circ} \mathrm{C}\right)$ & Canopy temperature $\left({ }^{\circ} \mathrm{C}\right)$ \\
\hline 1 & $51.62 \pm 3.25 \mathrm{a}$ & $34.24 \pm 1.46 \mathrm{hi}$ & $20.37 \pm 0.86 \mathrm{~cd}$ & $14.62 \pm 0.63 \mathrm{hi}$ \\
\hline 2 & $46.61 \pm 1.58 \mathrm{a}-\mathrm{c}$ & $32.89 \pm 0.16 \mathrm{ij}$ & $19.86 \pm 1.16 \mathrm{~d}$ & $15.23 \pm 0.05 \mathrm{e}-\mathrm{i}$ \\
\hline 3 & $36.76 \pm 1.45 \mathrm{a}-\mathrm{e}$ & $30.66 \pm 0.70 \mathrm{i}-1$ & $21.69 \pm 0.37 b-d$ & $13.38 \pm 0.23 \mathrm{i}$ \\
\hline 4 & $42.57 \pm 6.37 \mathrm{a}-\mathrm{d}$ & $34.54 \pm 0.72 \mathrm{hi}$ & $20.06 \pm 0.75 d$ & $16.07 \pm 0.82 b-h$ \\
\hline 5 & $33.96 \pm 4.37 \mathrm{~b}-\mathrm{e}$ & $30.13 \pm 1.79 \mathrm{i}-1$ & $21.04 \pm 0.65 \mathrm{~cd}$ & $16.29 \pm 0.97 b-h$ \\
\hline 6 & $24.58 \pm 8.63 \mathrm{e}$ & $30.72 \pm 1.59 \mathrm{i}-1$ & $22.40 \pm 0.96 a-d$ & $14.69 \pm 0.27 \mathrm{~g}-\mathrm{i}$ \\
\hline 7 & $48.46 \pm 6.84 a-c$ & $39.49 \pm 1.26 \mathrm{~g}$ & $20.12 \pm 0.44 d$ & $15.53 \pm 0.75 \mathrm{~d}-\mathrm{h}$ \\
\hline 8 & $42.03 \pm 4.63 \mathrm{a}-\mathrm{e}$ & $34.62 \pm 1.60 \mathrm{hi}$ & $20.84 \pm 0.47 \mathrm{~cd}$ & $15.81 \pm 0.28 \mathrm{c}-\mathrm{h}$ \\
\hline 9 & $34.19 \pm 2.91 \mathrm{a}-\mathrm{e}$ & $43.39 \pm 0.34 \mathrm{e}-\mathrm{g}$ & $20.47 \pm 1.29 \mathrm{~cd}$ & $15.11 \pm 0.48 \mathrm{f}-\mathrm{i}$ \\
\hline 10 & $50.26 \pm 3.75 \mathrm{ab}$ & $34.60 \pm 0.66 \mathrm{hi}$ & $20.84 \pm 1.22 \mathrm{~cd}$ & $14.78 \pm 0.85 \mathrm{~g}-\mathrm{i}$ \\
\hline 11 & $35.74 \pm 4.75 \mathrm{a}-\mathrm{e}$ & $38.82 \pm 2.03 \mathrm{gh}$ & $22.22 \pm 0.57 \mathrm{a}-\mathrm{d}$ & $14.83 \pm 0.57 \mathrm{~g}-\mathrm{i}$ \\
\hline 12 & $39.73 \pm 4.24 \mathrm{a}-\mathrm{e}$ & $27.06 \pm 3.39 \mathrm{kl}$ & $21.01 \pm 0.91 \mathrm{~cd}$ & $16.61 \pm 1.17 \mathrm{~b}-\mathrm{g}$ \\
\hline 13 & $35.35 \pm 2.32 \mathrm{a}-\mathrm{e}$ & $32.08 \pm 1.13 \mathrm{i}-\mathrm{k}$ & $20.32 \pm 0.71 \mathrm{~cd}$ & $17.22 \pm 0.27 b-d$ \\
\hline 14 & $24.56 \pm 7.04 \mathrm{e}$ & $39.96 \pm 1.68 \mathrm{fg}$ & $22.62 \pm 1.13 \mathrm{a}-\mathrm{d}$ & $16.83 \pm 1.23 b-f$ \\
\hline 15 & $41.55 \pm 0.74 a-e$ & $34.20 \pm 0.68 \mathrm{hi}$ & $20.81 \pm 1.01 \mathrm{~cd}$ & $15.81 \pm 0.28 \mathrm{c}-\mathrm{h}$ \\
\hline 16 & $39.90 \pm 1.66 \mathrm{a}-\mathrm{e}$ & $29.89 \pm 0.57 \mathrm{i}-1$ & $20.05 \pm 1.20 \mathrm{~d}$ & $16.31 \pm 0.60 b-h$ \\
\hline 17 & $37.42 \pm 2.42 \mathrm{a}-\mathrm{e}$ & $32.34 \pm 0.93 \mathrm{ij}$ & $22.28 \pm 1.53 \mathrm{a}-\mathrm{d}$ & $17.09 \pm 0.88 \mathrm{~b}-\mathrm{e}$ \\
\hline 18 & $33.51 \pm 13.90 \mathrm{~b}-\mathrm{e}$ & $34.48 \pm 1.10 \mathrm{hi}$ & $22.45 \pm 2.07 \mathrm{a}-\mathrm{d}$ & $17.87 \pm 0.69 b$ \\
\hline 19 & $35.33 \pm 3.52 \mathrm{a}-\mathrm{e}$ & $31.06 \pm 2.25 \mathrm{i}-1$ & $22.46 \pm 2.21 \mathrm{a}-\mathrm{d}$ & $16.48 \pm 0.48 b-h$ \\
\hline 20 & $34.17 \pm 6.44 a-e$ & $31.82 \pm 1.60 \mathrm{i}-\mathrm{k}$ & $22.11 \pm 1.18 \mathrm{a}-\mathrm{d}$ & $16.62 \pm 0.90 \mathrm{~b}-\mathrm{g}$ \\
\hline 21 & $38.53 \pm 4.70 \mathrm{a}-\mathrm{e}$ & $32.08 \pm 0.82 \mathrm{i}-\mathrm{k}$ & $21.27 \pm 0.60 \mathrm{~cd}$ & $17.58 \pm 0.64 b c$ \\
\hline 22 & $30.93 \pm 2.91 \mathrm{c}-\mathrm{e}$ & $26.22 \pm 1.501$ & $22.79 \pm 1.75 \mathrm{a}-\mathrm{d}$ & $16.92 \pm 0.67 b-f$ \\
\hline 23 & $49.57 \pm 2.46 \mathrm{ab}$ & $31.84 \pm 1.77 \mathrm{i}-\mathrm{k}$ & $20.21 \pm 1.08 \mathrm{~d}$ & $15.64 \pm 0.58 \mathrm{c}-\mathrm{h}$ \\
\hline 24 & $31.04 \pm 14.17 \mathrm{c}-\mathrm{e}$ & $28.77 \pm 1.61 \mathrm{j}-1$ & $21.86 \pm 2.43 \mathrm{a}-\mathrm{d}$ & $14.53 \pm 0.12 \mathrm{hi}$ \\
\hline 25 & $33.33 \pm 7.82 b-e$ & $26.62 \pm 1.411$ & $20.42 \pm 0.29 \mathrm{~cd}$ & $16.09 \pm 0.48 b c-h$ \\
\hline 26 & $40.07 \pm 1.33 \mathrm{a}-\mathrm{e}$ & $46.81 \pm 0.82 \mathrm{c}-\mathrm{e}$ & $22.13 \pm 0.52 \mathrm{a}-\mathrm{d}$ & $22.20 \pm 0.10 \mathrm{a}$ \\
\hline 27 & $34.53 \pm 3.25 \mathrm{a}-\mathrm{e}$ & $48.49 \pm 0.54 b-d$ & $20.93 \pm 0.37 \mathrm{~cd}$ & $21.48 \pm 0.19 \mathrm{a}$ \\
\hline 28 & $24.93 \pm 1.89 \mathrm{de}$ & $51.04 \pm 0.44 \mathrm{a}-\mathrm{c}$ & $20.63 \pm 0.18 \mathrm{~cd}$ & $21.48 \pm 0.18 \mathrm{a}$ \\
\hline 29 & $39.90 \pm 5.06 \mathrm{a}-\mathrm{e}$ & $52.67 \pm 3.08 \mathrm{ab}$ & $20.60 \pm 0.57 \mathrm{~cd}$ & $21.06 \pm 0.08 \mathrm{a}$ \\
\hline 30 & $40.93 \pm 1.93 \mathrm{a}-\mathrm{e}$ & $54.73 \pm 0.64 a$ & $22.87 \pm 1.43 \mathrm{a}-\mathrm{d}$ & $21.33 \pm 0.16 \mathrm{a}$ \\
\hline 31 & $31.37 \pm 0.52 \mathrm{c}-\mathrm{e}$ & $39.89 \pm 1.10 \mathrm{fg}$ & $20.13 \pm 0.37 d$ & $21.50 \pm 0.27 \mathrm{a}$ \\
\hline 32 & $35.30 \pm 1.10 \mathrm{a}-\mathrm{e}$ & $46.84 \pm 2.17 \mathrm{c}-\mathrm{e}$ & $20.90 \pm 0.27 \mathrm{~cd}$ & $20.88 \pm 0.13 a$ \\
\hline 33 & $31.13 \pm 1.07 \mathrm{c}-\mathrm{e}$ & $44.98 \pm 1.92 \mathrm{de}$ & $23.77 \pm 1.35 \mathrm{a}-\mathrm{c}$ & $20.41 \pm 0.15 a$ \\
\hline 34 & $41.60 \pm 3.40 \mathrm{a}-\mathrm{e}$ & $47.89 \pm 1.78 \mathrm{c}-\mathrm{e}$ & $24.73 \pm 1.16 \mathrm{ab}$ & $20.72 \pm 0.04 a$ \\
\hline 35 & $43.63 \pm 5.17 \mathrm{a}-\mathrm{c}$ & $44.46 \pm 1.56 \mathrm{~d}-\mathrm{f}$ & $23.77 \pm 0.52 \mathrm{a}-\mathrm{c}$ & $20.63 \pm 0.07 \mathrm{a}$ \\
\hline 36 & $39.97 \pm 5.81 \mathrm{a}-\mathrm{e}$ & $21.37 \pm 0.91 \mathrm{~m}$ & $25.23 \pm 1.24 \mathrm{a}$ & $21.64 \pm 0.36 \mathrm{a}$ \\
\hline Average & 37.64 & 36.71 & 21.56 & 17.54 \\
\hline $\mathrm{CV} / \%$ & 18.07 & 22.61 & 6.34 & 15.10 \\
\hline
\end{tabular}

Data presented are the means \pm SE of three independent experiments. Different lowercase letters indicate that there is significant difference $(\mathrm{p}<0.05)$ between the different varieties of the same treatment. 
Table 2. Effects of saline-alkali stress and drought stress on stay-green ability, net photosynthetic rate $\left(P_{\mathrm{n}}, \mu \mathrm{mol} \cdot \mathrm{m}^{-2} \cdot \mathrm{s}^{-1}\right)$, stomatal conductance $\left(G_{\mathrm{s}}, \mathrm{mol} / \mathrm{m}^{2} / \mathrm{s}\right)$, and Transpiration rate $\left(T_{r}, \mathrm{mmol} / \mathrm{m}^{2} / \mathrm{s}\right)$ in maize varieties.

\begin{tabular}{|c|c|c|c|c|}
\hline & Saline alkali & Drought land & Saline alkali & Drought land \\
\hline Number & Stay-green ability & Stay-green ability & $\begin{array}{l}\text { Net photosynthetic rate } \\
\left(\mu \mathrm{mol} / \mathrm{m}^{2} / \mathrm{s}\right)\end{array}$ & $\begin{array}{l}\text { Net photosynthetic rate } \\
\left(\mu \mathrm{mol} / \mathrm{m}^{2} / \mathrm{s}\right)\end{array}$ \\
\hline 1 & $0.77 \pm 0.03 \mathrm{ab}$ & $0.57 \pm 0.04 \mathrm{~cd}$ & $14.59 \pm 1.78 \mathrm{ab}$ & $9.62 \pm 0.03 \mathrm{i}-\mathrm{n}$ \\
\hline 2 & $0.79 \pm 0.01 \mathrm{a}$ & $0.60 \pm 0.01 b c$ & $13.02 \pm 1.40 \mathrm{ab}$ & $13.59 \pm 0.34 b-d$ \\
\hline 3 & $0.73 \pm 0.03 a-d$ & $0.43 \pm 0.04 \mathrm{e}-\mathrm{h}$ & $14.07 \pm 1.73 \mathrm{ab}$ & $11.55 \pm 0.23 \mathrm{c}-\mathrm{k}$ \\
\hline 4 & $0.72 \pm 0.05 a-d$ & $0.46 \pm 0.04 \mathrm{~d}-\mathrm{h}$ & $12.55 \pm 1.39 \mathrm{ab}$ & $11.86 \pm 0.17 \mathrm{c}-\mathrm{j}$ \\
\hline 5 & $0.72 \pm 0.02 \mathrm{a}-\mathrm{d}$ & $0.41 \pm 0.07 \mathrm{e}-\mathrm{h}$ & $13.09 \pm 1.66 \mathrm{ab}$ & $12.75 \pm 0.52 b-g$ \\
\hline 6 & $0.51 \pm 0.14 \mathrm{f}-\mathrm{j}$ & $0.49 \pm 0.04 \mathrm{c}-\mathrm{g}$ & $14.93 \pm 2.05 \mathrm{ab}$ & $13.59 \pm 0.74 b-d$ \\
\hline 7 & $0.71 \pm 0.07 \mathrm{a}-\mathrm{d}$ & $0.41 \pm 0.06 \mathrm{e}-\mathrm{h}$ & $16.03 \pm 3.45 \mathrm{a}$ & $16.87 \pm 0.86 \mathrm{a}$ \\
\hline 8 & $0.70 \pm 0.02 \mathrm{a}-\mathrm{d}$ & $0.54 \pm 0.05 \mathrm{c}-\mathrm{e}$ & $12.58 \pm 0.59 \mathrm{ab}$ & $11.26 \pm 0.45 \mathrm{~d}-\mathrm{k}$ \\
\hline 9 & $0.60 \pm 0.03 c-i$ & $0.33 \pm 0.04 \mathrm{~h}$ & $13.46 \pm 2.77 \mathrm{ab}$ & $13.90 \pm 0.46 b c$ \\
\hline 10 & $0.75 \pm 0.03 a-c$ & $0.50 \pm 0.02 \mathrm{c}-\mathrm{g}$ & $11.29 \pm 1.04 \mathrm{a}-\mathrm{c}$ & $11.63 \pm 0.42 \mathrm{c}-\mathrm{k}$ \\
\hline 11 & $0.70 \pm 0.02 \mathrm{a}-\mathrm{d}$ & $0.51 \pm 0.06 c-f$ & $13.93 \pm 2.24 \mathrm{ab}$ & $10.88 \pm 0.18 \mathrm{f}-\mathrm{k}$ \\
\hline 12 & $0.71 \pm 0.03 \mathrm{a}-\mathrm{d}$ & $0.34 \pm 0.02 \mathrm{~h}$ & $12.02 \pm 1.51 \mathrm{a}-\mathrm{c}$ & $11.06 \pm 0.47 \mathrm{e}-\mathrm{k}$ \\
\hline 13 & $0.68 \pm 0.03 a-e$ & $0.35 \pm 0.08 \mathrm{~h}$ & $12.61 \pm 1.13 \mathrm{ab}$ & $11.09 \pm 0.52 \mathrm{~d}-\mathrm{k}$ \\
\hline 14 & $0.58 \pm 0.12 \mathrm{~d}-\mathrm{j}$ & $0.54 \pm 0.01 \mathrm{c}-\mathrm{e}$ & $12.26 \pm 1.54 \mathrm{ab}$ & $10.45 \pm 0.34 \mathrm{~g}-1$ \\
\hline 15 & $0.71 \pm 0.01 \mathrm{a}-\mathrm{d}$ & $0.53 \pm 0.04 c-f$ & $11.26 \pm 0.86 \mathrm{a}-\mathrm{c}$ & $13.58 \pm 1.03 b-d$ \\
\hline 16 & $0.72 \pm 0.03 a-d$ & $0.52 \pm 0.04 c-f$ & $9.74 \pm 0.78 b c$ & $12.17 \pm 0.42 b-h$ \\
\hline 17 & $0.67 \pm 0.03 \mathrm{a}-\mathrm{e}$ & $0.54 \pm 0.03 \mathrm{c}-\mathrm{e}$ & $11.01 \pm 0.26 \mathrm{a}-\mathrm{c}$ & $13.30 \pm 0.30 b-f$ \\
\hline 18 & $0.62 \pm 0.11 b-h$ & $0.43 \pm 0.03 \mathrm{e}-\mathrm{h}$ & $12.34 \pm 2.11 \mathrm{ab}$ & $13.49 \pm 0.22 b-e$ \\
\hline 19 & $0.58 \pm 0.04 \mathrm{~d}-\mathrm{j}$ & $0.43 \pm 0.03 \mathrm{e}-\mathrm{h}$ & $15.98 \pm 1.68 \mathrm{a}$ & $12.72 \pm 0.55 \mathrm{~b}-\mathrm{g}$ \\
\hline 20 & $0.68 \pm 0.04 a-e$ & $0.40 \pm 0.08 \mathrm{f}-\mathrm{h}$ & $12.24 \pm 0.75 \mathrm{ab}$ & $13.13 \pm 0.29 b-f$ \\
\hline 21 & $0.66 \pm 0.05 a-f$ & $0.58 \pm 0.03 \mathrm{~cd}$ & $13.57 \pm 1.66 \mathrm{ab}$ & $13.52 \pm 0.76 \mathrm{~b}-\mathrm{e}$ \\
\hline 22 & $0.69 \pm 0.02 \mathrm{a}-\mathrm{e}$ & $0.37 \pm 0.02 \mathrm{gh}$ & $12.37 \pm 1.47 \mathrm{ab}$ & $12.07 \pm 0.25 \mathrm{c}-\mathrm{i}$ \\
\hline 23 & $0.77 \pm 0.02 \mathrm{ab}$ & $0.46 \pm 0.06 \mathrm{c}-\mathrm{h}$ & $14.55 \pm 1.89 \mathrm{ab}$ & $11.93 \pm 0.24 c-i$ \\
\hline 24 & $0.57 \pm 0.03 \mathrm{~d}-\mathrm{j}$ & $0.57 \pm 0.01 \mathrm{~cd}$ & $11.14 \pm 1.31 \mathrm{a}-\mathrm{c}$ & $14.60 \pm 0.37 b$ \\
\hline 25 & $0.63 \pm 0.03 a-g$ & $0.60 \pm 0.03 b-d$ & $13.62 \pm 1.91 \mathrm{ab}$ & $11.16 \pm 0.36 \mathrm{~d}-\mathrm{k}$ \\
\hline 26 & $0.61 \pm 0.03 b-h$ & $0.76 \pm 0.09 \mathrm{a}$ & $7.16 \pm 1.33 \mathrm{~cd}$ & $7.69 \pm 0.31 \mathrm{~m}-\mathrm{p}$ \\
\hline 27 & $0.44 \pm 0.03 \mathrm{ij}$ & $0.81 \pm 0.00 \mathrm{a}$ & $3.43 \pm 0.14 \mathrm{~d}$ & $8.25 \pm 0.391-\mathrm{o}$ \\
\hline 28 & $0.46 \pm 0.04 h-j$ & $0.82 \pm 0.03 \mathrm{a}$ & $2.29 \pm 0.20 \mathrm{~d}$ & $9.95 \pm 1.51 \mathrm{~h}-\mathrm{m}$ \\
\hline 29 & $0.49 \pm 0.04 \mathrm{~g}-\mathrm{j}$ & $0.81 \pm 0.03 \mathrm{a}$ & $3.96 \pm 0.28 \mathrm{~d}$ & $6.77 \pm 0.67 \mathrm{op}$ \\
\hline 30 & $0.51 \pm 0.04 f-j$ & $0.75 \pm 0.00 \mathrm{a}$ & $4.82 \pm 2.42 \mathrm{~d}$ & $7.31 \pm 2.21 \mathrm{n}-\mathrm{p}$ \\
\hline 31 & $0.43 \pm 0.04 \mathrm{j}$ & $0.71 \pm 0.02 \mathrm{ab}$ & $3.13 \pm 1.35 \mathrm{~d}$ & $9.76 \pm 0.47 \mathrm{~h}-\mathrm{m}$ \\
\hline 32 & $0.43 \pm 0.04 \mathrm{j}$ & $0.74 \pm 0.02 \mathrm{a}$ & $2.24 \pm 0.71 \mathrm{~d}$ & $9.36 \pm 1.02 \mathrm{k}-\mathrm{n}$ \\
\hline 33 & $0.49 \pm 0.05 \mathrm{~g}-\mathrm{j}$ & $0.75 \pm 0.04 a$ & $10.35 \pm 0.11 b c$ & $13.03 \pm 0.37 b-f$ \\
\hline 34 & $0.54 \pm 0.01 e-j$ & $0.79 \pm 0.02 \mathrm{a}$ & $2.42 \pm 0.16 \mathrm{~d}$ & $9.42 \pm 1.07 \mathrm{j}-\mathrm{n}$ \\
\hline 35 & $0.65 \pm 0.04 a-f$ & $0.78 \pm 0.03 \mathrm{a}$ & $4.11 \pm 0.21 d$ & $9.82 \pm 1.66 \mathrm{~h}-\mathrm{m}$ \\
\hline 36 & $0.47 \pm 0.02 \mathrm{~h}-\mathrm{j}$ & $0.59 \pm 0.01 b-d$ & $13.03 \pm 1.84 \mathrm{ab}$ & $5.76 \pm 0.61 p$ \\
\hline Average & 0.63 & 0.56 & 10.59 & 11.36 \\
\hline $\mathrm{CV} / \%$ & 17.34 & 27.08 & 40.63 & 20.92 \\
\hline
\end{tabular}


(Contd.)

\begin{tabular}{|c|c|c|c|c|}
\hline \multirow[b]{2}{*}{ Number } & Saline alkali & Drought land & Saline alkali & Drought land \\
\hline & $\begin{array}{l}\text { Stomatal conductance } \\
\left(\mathrm{mol} / \mathrm{m}^{2} / \mathrm{s}\right)\end{array}$ & $\begin{array}{l}\text { Stomatal conductance } \\
\left(\mathrm{mol} / \mathrm{m}^{2} / \mathrm{s}\right)\end{array}$ & $\begin{array}{l}\text { Transpiration rate } \\
\left(\mathrm{mmol} / \mathrm{m}^{2} / \mathrm{s}\right)\end{array}$ & $\begin{array}{l}\text { Transpiration rate } \\
\left(\mathrm{mmol} / \mathrm{m}^{2} / \mathrm{s}\right)\end{array}$ \\
\hline 1 & $0.10 \pm 0.02 b$ & $0.03 \pm 0.00 \mathrm{i}-1$ & $2.60 \pm 0.43 b$ & $1.16 \pm 0.00 f-1$ \\
\hline 2 & $0.07 \pm 0.01 b$ & $0.05 \pm 0.01 \mathrm{~d}-\mathrm{j}$ & $1.85 \pm 0.28 b$ & $1.65 \pm 0.14 b c$ \\
\hline 3 & $0.11 \pm 0.03 b$ & $0.04 \pm 0.00 \mathrm{e}-1$ & $2.76 \pm 0.46 \mathrm{~b}$ & $1.43 \pm 0.02 \mathrm{c}-\mathrm{g}$ \\
\hline 4 & $0.08 \pm 0.01 \mathrm{~b}$ & $0.04 \pm 0.00 \mathrm{e}-1$ & $2.21 \pm 0.60 \mathrm{~b}$ & $1.36 \pm 0.02 \mathrm{~d}-\mathrm{h}$ \\
\hline 5 & $0.09 \pm 0.02 b$ & $0.05 \pm 0.00 \mathrm{~d}-\mathrm{k}$ & $2.26 \pm 0.39 b$ & $1.46 \pm 0.03 \mathrm{c}-\mathrm{e}$ \\
\hline 6 & $0.08 \pm 0.01 \mathrm{~b}$ & $0.06 \pm 0.00 \mathrm{~b}-\mathrm{e}$ & $2.04 \pm 0.18 \mathrm{~b}$ & $1.76 \pm 0.08 b$ \\
\hline 7 & $0.11 \pm 0.03 b$ & $0.07 \pm 0.01 \mathrm{ab}$ & $3.04 \pm 1.13 \mathrm{~b}$ & $2.07 \pm 0.13 \mathrm{a}$ \\
\hline 8 & $0.13 \pm 0.06 b$ & $0.07 \pm 0.00 \mathrm{ab}$ & $3.58 \pm 1.70 \mathrm{~b}$ & $2.07 \pm 0.09 \mathrm{a}$ \\
\hline 9 & $0.08 \pm 0.02 b$ & $0.05 \pm 0.00 \mathrm{c}-\mathrm{g}$ & $2.12 \pm 0.46 b$ & $1.56 \pm 0.07 \mathrm{~b}-\mathrm{d}$ \\
\hline 10 & $0.09 \pm 0.04 \mathrm{~b}$ & $0.04 \pm 0.00 \mathrm{~h}-1$ & $2.54 \pm 1.11 \mathrm{~b}$ & $1.09 \pm 0.05 \mathrm{~h}-\mathrm{n}$ \\
\hline 11 & $0.08 \pm 0.02 b$ & $0.04 \pm 0.00 \mathrm{~g}-1$ & $2.07 \pm 0.49 \mathrm{~b}$ & $1.16 \pm 0.00 \mathrm{f}-1$ \\
\hline 12 & $0.07 \pm 0.02 b$ & $0.04 \pm 0.00 \mathrm{f}-1$ & $1.86 \pm 0.31 \mathrm{~b}$ & $1.17 \pm 0.04 \mathrm{f}-1$ \\
\hline 13 & $0.09 \pm 0.01 \mathrm{~b}$ & $0.05 \pm 0.00 \mathrm{~d}-\mathrm{j}$ & $2.43 \pm 0.43 b$ & $1.34 \pm 0.05 \mathrm{~d}-\mathrm{h}$ \\
\hline 14 & $0.11 \pm 0.03 b$ & $0.03 \pm 0.00 \mathrm{kl}$ & $2.69 \pm 0.57 b$ & $0.95 \pm 0.04 \mathrm{j}-\mathrm{o}$ \\
\hline 15 & $0.14 \pm 0.08 b$ & $0.06 \pm 0.00 \mathrm{a}-\mathrm{d}$ & $3.15 \pm 1.20 \mathrm{~b}$ & $1.68 \pm 0.08 b c$ \\
\hline 16 & $0.12 \pm 0.05 b$ & $0.04 \pm 0.00 \mathrm{e}-1$ & $2.90 \pm 0.87 b$ & $1.18 \pm 0.07 \mathrm{e}-1$ \\
\hline 17 & $0.18 \pm 0.11 \mathrm{ab}$ & $0.04 \pm 0.00 \mathrm{e}-1$ & $3.74 \pm 1.54 b$ & $1.16 \pm 0.04 f-1$ \\
\hline 18 & $0.13 \pm 0.07 b$ & $0.05 \pm 0.00 \mathrm{e}-\mathrm{k}$ & $3.02 \pm 1.07 \mathrm{~b}$ & $1.23 \pm 0.04 \mathrm{e}-\mathrm{j}$ \\
\hline 19 & $0.09 \pm 0.02 b$ & $0.05 \pm 0.01 \mathrm{e}-\mathrm{k}$ & $2.33 \pm 0.37 b$ & $1.20 \pm 0.14 \mathrm{e}-\mathrm{k}$ \\
\hline 20 & $0.16 \pm 0.06 \mathrm{~b}$ & $0.05 \pm 0.00 \mathrm{~d}-\mathrm{i}$ & $3.38 \pm 0.70 \mathrm{~b}$ & $1.35 \pm 0.02 \mathrm{~d}-\mathrm{h}$ \\
\hline 21 & $0.14 \pm 0.04 b$ & $0.04 \pm 0.00 \mathrm{f}-1$ & $3.52 \pm 1.23 b$ & $1.07 \pm 0.09 \mathrm{~h}-\mathrm{n}$ \\
\hline 22 & $0.11 \pm 0.05 b$ & $0.03 \pm 0.00 \mathrm{j}-1$ & $2.69 \pm 0.99 b$ & $0.92 \pm 0.07 \mathrm{k}-\mathrm{o}$ \\
\hline 23 & $0.09 \pm 0.02 b$ & $0.04 \pm 0.01 \mathrm{e}-\mathrm{k}$ & $2.40 \pm 0.43 b$ & $1.03 \pm 0.05 \mathrm{i}-\mathrm{n}$ \\
\hline 24 & $0.07 \pm 0.01 \mathrm{~b}$ & $0.06 \pm 0.00 \mathrm{~b}-\mathrm{f}$ & $1.85 \pm 0.17 b$ & $1.24 \pm 0.07 \mathrm{e}-\mathrm{i}$ \\
\hline 25 & $0.08 \pm 0.02 b$ & $0.05 \pm 0.00 \mathrm{e}-\mathrm{k}$ & $2.34 \pm 0.64 b$ & $1.16 \pm 0.08 f-1$ \\
\hline 26 & $0.10 \pm 0.02 b$ & $0.04 \pm 0.00 \mathrm{e}-\mathrm{k}$ & $2.89 \pm 0.50 \mathrm{~b}$ & $1.01 \pm 0.02 \mathrm{i}-\mathrm{n}$ \\
\hline 27 & $0.08 \pm 0.01 \mathrm{~b}$ & $0.05 \pm 0.00 \mathrm{~d}-\mathrm{h}$ & $2.35 \pm 0.33 b$ & $1.15 \pm 0.01 \mathrm{~g}-\mathrm{m}$ \\
\hline 28 & $0.07 \pm 0.01 \mathrm{~b}$ & $0.07 \pm 0.01 \mathrm{a}-\mathrm{c}$ & $2.05 \pm 0.13 b$ & $1.44 \pm 0.13 c-f$ \\
\hline 29 & $0.10 \pm 0.01 \mathrm{~b}$ & $0.04 \pm 0.01 \mathrm{~h}-1$ & $2.74 \pm 0.29 b$ & $0.85 \pm 0.12 \mathrm{no}$ \\
\hline 30 & $0.13 \pm 0.06 b$ & $0.04 \pm 0.01 \mathrm{e}-1$ & $3.24 \pm 1.20 \mathrm{~b}$ & $0.91 \pm 0.221-\mathrm{o}$ \\
\hline 31 & $0.15 \pm 0.03 b$ & $0.07 \pm 0.00 \mathrm{a}$ & $3.66 \pm 0.56 b$ & $1.44 \pm 0.09 c-f$ \\
\hline 32 & $0.18 \pm 0.04 \mathrm{ab}$ & $0.04 \pm 0.01 \mathrm{e}-1$ & $4.14 \pm 0.76 \mathrm{ab}$ & $0.90 \pm 0.171-\mathrm{o}$ \\
\hline 33 & $0.13 \pm 0.00 \mathrm{~b}$ & $0.07 \pm 0.00 \mathrm{a}-\mathrm{c}$ & $3.23 \pm 0.02 b$ & $1.25 \pm 0.03 \mathrm{e}-\mathrm{i}$ \\
\hline 34 & $0.09 \pm 0.01 \mathrm{~b}$ & $0.04 \pm 0.01 \mathrm{~h}-1$ & $2.37 \pm 0.16 b$ & $0.82 \pm 0.09 \mathrm{no}$ \\
\hline 35 & $0.08 \pm 0.00 \mathrm{~b}$ & $0.04 \pm 0.01 \mathrm{~g}-1$ & $2.21 \pm 0.02 b$ & $0.87 \pm 0.17 \mathrm{~m}-\mathrm{o}$ \\
\hline 36 & $0.29 \pm 0.04 \mathrm{a}$ & $0.03 \pm 0.001$ & $6.10 \pm 0.56 \mathrm{a}$ & $0.69 \pm 0.01 \mathrm{o}$ \\
\hline Average & 0.11 & 0.05 & 2.79 & 1.24 \\
\hline $\mathrm{CV} / \%$ & 39.51 & 23.91 & 29.36 & 26.10 \\
\hline
\end{tabular}

Data presented are the means \pm SE of three independent experiment. Different lowercase letters indicate that there is significant difference $(\mathrm{p}<0.05)$ between the different varieties of the same treatment. 
number, axis diameter and ear row number. Significant negative correlations were observed between dry fresh ratio and plant height, SPAD value, above ground biomass, canopy temperature and the stem diameter, net photosynthetic rate.

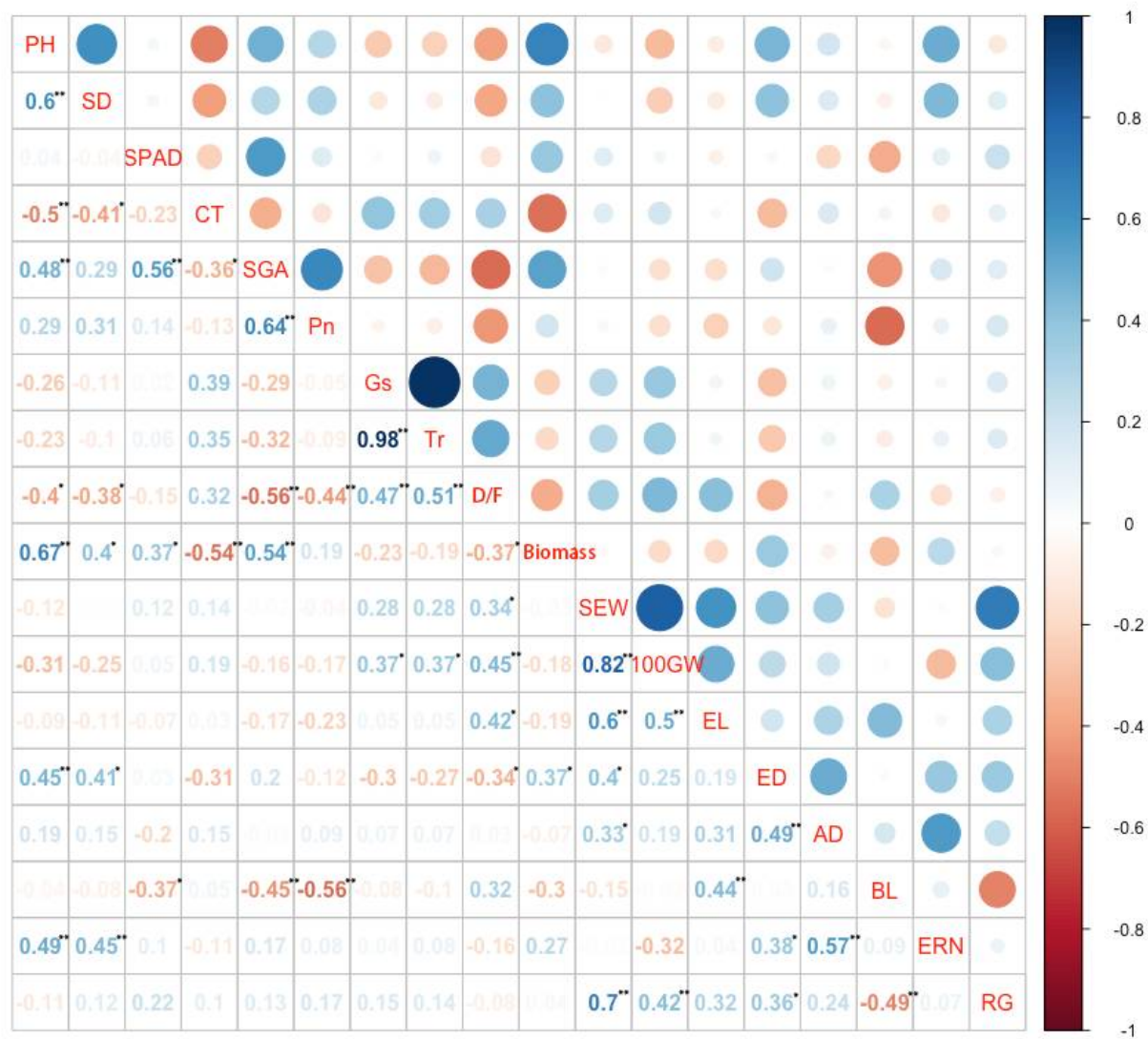

Fig. 1. The correlations between agronomic index, photosynthetic parameters and yield indicees under saline alkali condition.

The color shade and size of the circle in the upper right corner represent the strength of the correlation. The size of the figure in the lower left corner represents the size of the correlation coefficient, *represents a significant correlation (p $<0.05)$, and **represents a highly significant correlation $(\mathrm{p}<0.01)$. PH - Plant height, SD - Stem diameter, SPAD Relative chlorophyll content, CT - Canopy temperature, SGA - Stay-green ability, Pn - Net photosynthetic rate, GsStomatal conductance, $\mathrm{Tr}$ - Transpiration rate, D/F- The ratio of dry weight and fresh weight, Biomass- Aboveground biomass, SEW - Single ear weight, 100GW - 100-Grain Weight, EL- Ear length, ED - Ear diameter, AD - Axis diameter, BL - Bald length, ERN - Ear row number and RG - Row grains.

According to the correlation analysis of each index in different maize varieties, there were significant differences among different indicators, the information they provide were overlapped, and the variation range of each index was different, which played a different role in the tolerance evaluation. The drought tolerance and saline-alkali tolerance of maize are a complex colligation character, which are the combined effect of several factors. Use of a single index cannot accurately evaluate the drought tolerance and saline-alkali tolerance of each varieties. In order to make a more accurate and comprehensive evaluation the drought and saline-alkali tolerant of the maize varieties at mature stage is important. Workers should take various aspects into consideration rather than only use a single index to reflect the salt and drought tolerance of plants. Principal 
component analysis (PCA) and subordination function analysis (SFA) were used to comprehensively evaluate and classify the salt tolerance and drought tolerance of maize varieties. According to the eigenvalue and the cumulative contribution rate of each index to filter the main components, and then make a comprehensive evaluation for varietal tolerancy ability.

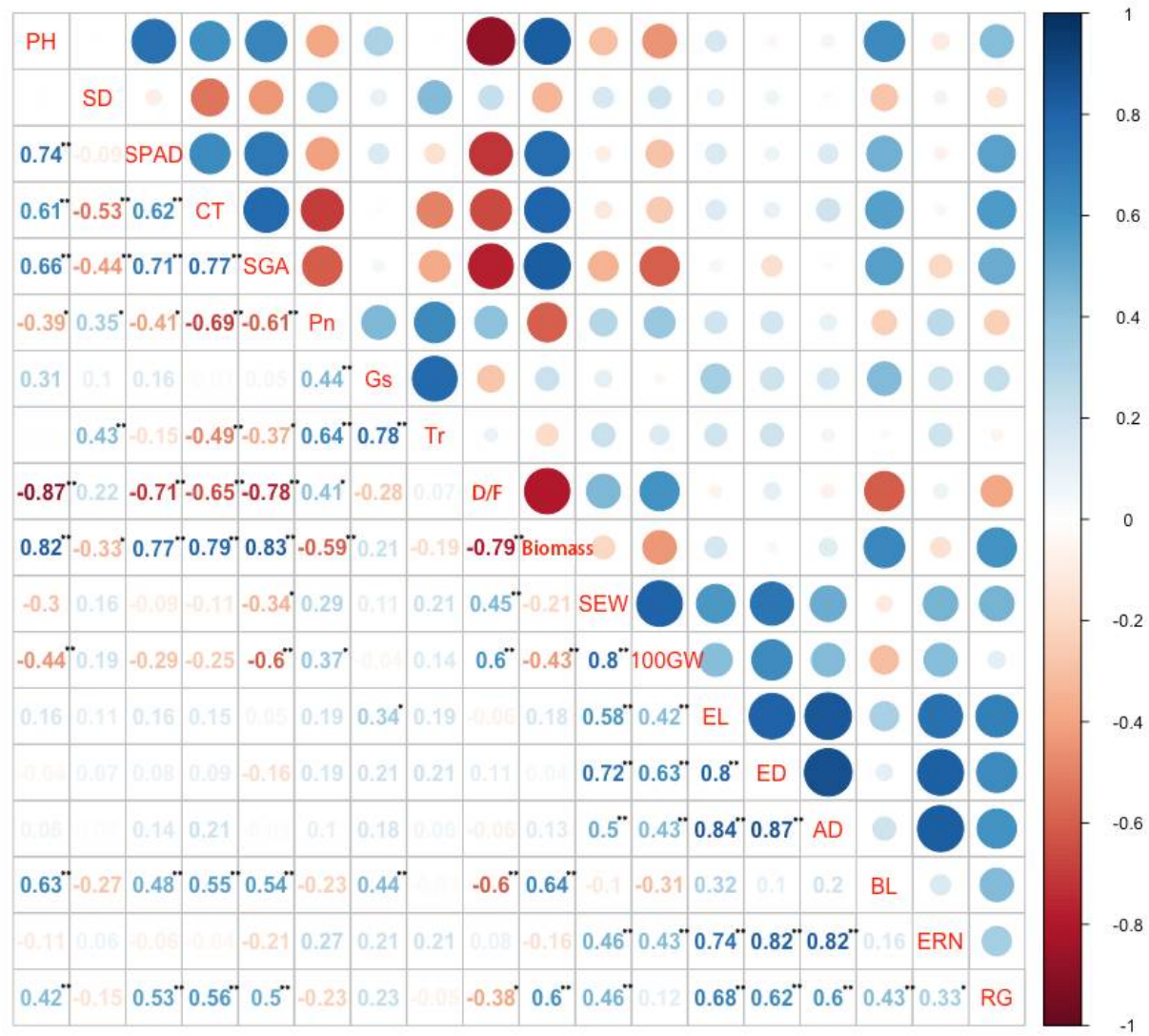

Fig. 2. The correlations between agronomic index, photosynthetic parameters and yield indicators under dry land condition. The color shade and size of the circle in the upper right corner represent the strength of the correlation. The size of the figure in the lower left corner represents the size of the correlation coefficient, *represents a significant correlation $(\mathrm{p}<0.05)$, and **represents a highly significant correlation $(\mathrm{p}<0.01)$. PH - Plant height, SD - Stem diameter, SPAD - Relative chlorophyll content, CT - Canopy temperature, SGA - Stay-green ability, Pn - Net photosynthetic rate, Gs - Stomatal conductance, $\mathrm{Tr}$ - Transpiration rate, D/F - The ratio of dry weight and fresh weight, Biomass - Aboveground biomass, SEW - Single ear weight, 100GW - 100-Grain weight, EL- Ear length, ED - Ear diameter, AD - Axis diameter, BL - Bald length, ERN - Ear row number and RG - Row grains.

The identification of drought tolerance and saline-alkali tolerance in maize varieties is a complicated process which is caused by many factors. It is difficult to evaluate drought tolerance and saline-alkali tolerance comprehensively and accurately by only one single indicator, so the use of the subordinate function method can be used. As shown in Table 3, based on the subordinate function values of 18 indices, 18 single indices were transformed into 5 principal components, and the composite score of each maize variety (lines) was calculated by principal component analysis (PCA). The Eigen values of the population from first to fifth reached over 1, and the first five principal components of the cumulative contribution rate accounted for $76.433 \%$, so it may be considered to retain the first five principal ingredients. 
Table 3. Eigen values, cumulative contribution rate of principal components and Eigen vector matrix by principal component analysis of different maize varieties under saline-alkali condition.

\begin{tabular}{lrrrrr}
\hline & $C I 1$ & $C I 2$ & $C I 3$ & $C I 4$ & $C I 5$ \\
\hline X1 & 0.39 & 0.08 & 0.16 & -0.07 & 0.28 \\
X2 & 0.15 & 0.01 & 0.15 & -0.39 & 0.17 \\
X3 & 0.35 & 0.06 & -0.18 & 0.15 & -0.28 \\
X4 & -0.35 & -0.19 & 0.23 & 0.05 & 0.15 \\
X5 & 0.38 & 0.07 & -0.19 & 0.20 & -0.27 \\
X6 & -0.05 & -0.17 & -0.20 & -0.16 & 0.47 \\
X7 & 0.04 & -0.05 & 0.51 & 0.33 & -0.08 \\
X8 & 0.12 & -0.03 & 0.51 & 0.30 & 0.02 \\
X9 & -0.05 & 0.20 & -0.06 & 0.26 & 0.46 \\
X10 & 0.36 & 0.24 & 0.11 & 0.08 & 0.17 \\
X11 & -0.15 & 0.46 & -0.03 & 0.11 & 0.10 \\
X12 & -0.22 & 0.33 & 0.00 & 0.33 & 0.12 \\
X13 & -0.16 & 0.38 & -0.10 & -0.01 & -0.21 \\
X14 & -0.01 & 0.42 & 0.19 & -0.15 & -0.02 \\
X15 & -0.16 & 0.14 & 0.33 & -0.35 & 0.22 \\
X16 & -0.28 & -0.11 & 0.16 & -0.02 & -0.40 \\
X17 & 0.27 & 0.05 & 0.26 & -0.37 & -0.09 \\
X18 & -0.10 & 0.39 & -0.08 & -0.27 & -0.13 \\
Eigen values & 4.84 & 3.28 & 2.46 & 1.82 & 1.36 \\
Cumulative & 26.90 & 45.09 & 58.76 & 68.89 & 76.43 \\
contribution rate/\% & & & & \\
\hline
\end{tabular}

X1: Plant height, X2: Stem diameter, X3: SPAD values, X4: Canopy temperature, X5: Stay-green ability, X6: Net photosynthetic rate, X7: Stomatal conductance, X8: Transpiration rate, X9: The ratio of dry weight and fresh weight, X10: Aboveground biomass, X11: Single ear weight, X12:Hundred-grain weight, X13: Ear length, X14: Ear diameter, X15: Axis diameter, X16: Bald length, X17: Ear row number, X18: Row grains.

Through further analysis of the PCA's results by membership function method, the results showed that the tolerance to saline-alkali of 36 maize varieties was different (Table 4). According to the comprehensive evaluation value (D value), the line of 133-2/1528 was the most saline-alkali tolerant, and Ke Duo 8 had the weakest saline-alkali tolerance, other varieties are between the two. Selecting the comprehensive evaluation value (D value) under saline-alkali treatment, the results of systematic cluster analysis (Fig. 3) showed that the 36 maize varieties can be divided into four groups. Three materials (the number of 7, 13 and 16) included in the first group, belong to highly saline-alkali tolerant. Ten materials (the number of 12, 22, 3, 18, 36, 4, 17, 8, 10 and 26) included in the second group belong to moderately saline-alkali tolerant. Nineteen materials (the number of $23,31,11,20,2,21,9,6,5,32,15,29,33,35,19,27,34,14$ and 30) included in the third group belong to saline-alkali sensitive. Four materials (the number of 1,24, 25 and 28) included in the fourth group belong to extremely saline-alkali sensitive. The comprehensive D value of the first group was the highest, the second group was the second, and the fourth group was the least. By comparing the results of comprehensive D value and cluster analysis, it showed that the cluster analysis can scientifically regard the 36 maize varieties be divided into four groups that had different degrees of saline-alkali tolerance, and this can provide guidance for further experiments. 


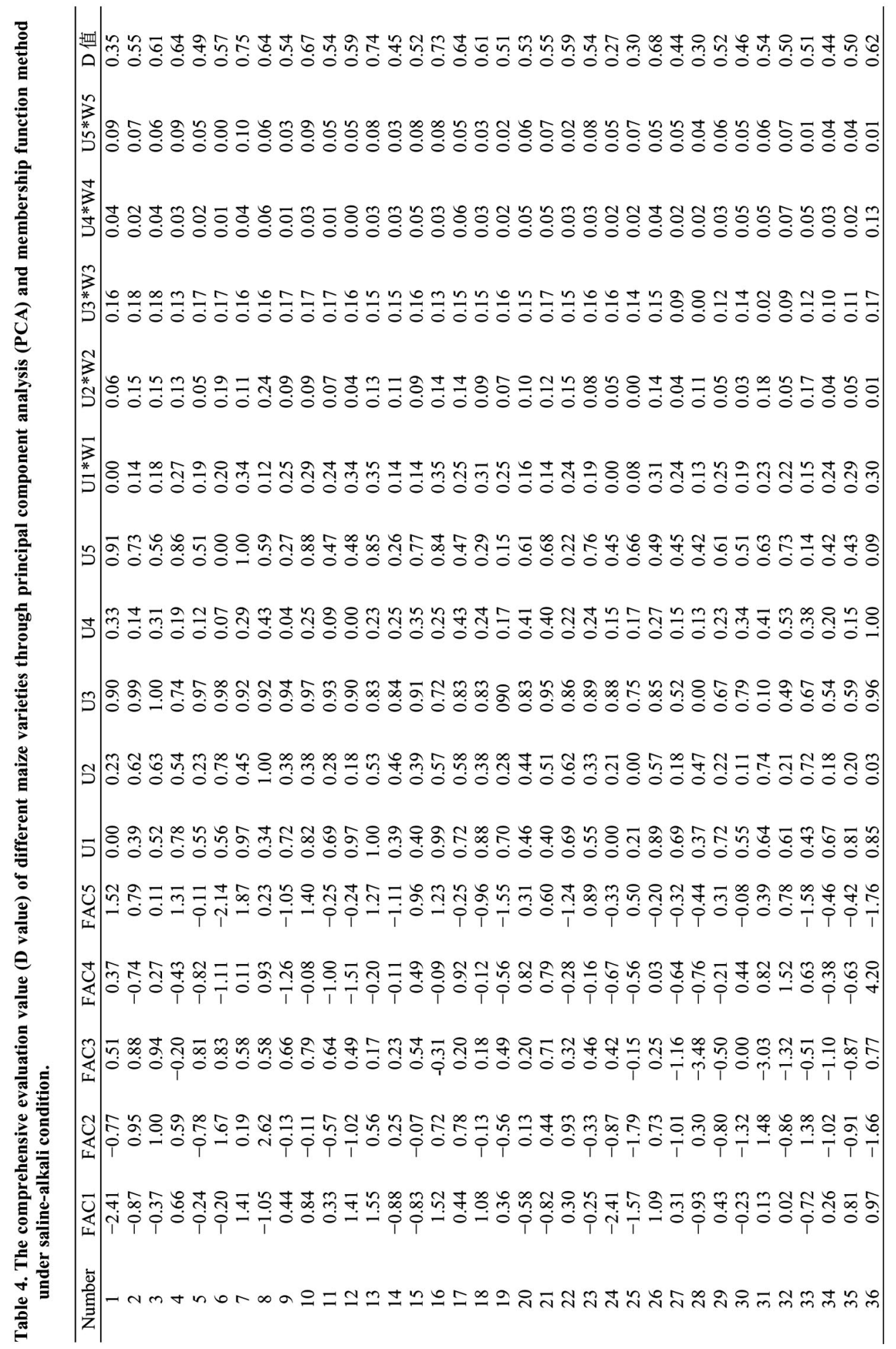


According to the formula in 2.4 data analysis, the membership function values ( $U$ value) of 36 maize varieties were calculated respectively, and the weight of each index was $0.414,0.333$, 0.153 and 0.100 , respectively. Finally, the comprehensive D value was calculated. The specific results as Tables 5 and 6 showed, D value can reflect the drought-tolerant ability of plants. Combined with Fig. 4, 36 maize varieties can be divided into five groups under dry land conditions. Two materials (the number of 28 and 31) included in the first group belong to highly drought tolerant. Fourteen materials (the number of 15, 26, 33, 6, 32, 29, 35, 34, 14, 27, 8, 7, 30 and 16) included in the second group belong to medium drought tolerant. Fifteen materials (the number of $2,13,17,10,19,5,20,9,3,11,21,25,18,12$ and 4) in the third group belong to weak drought tolerant. Eight materials (the number of 22, 23, 24 and 36) included in the fourth group belong to drought sensitive. The number of 1 included in the fifth group belongs to extremely drought sensitive.

Table 5. Eigenvalues, cumulative contribution rate of principal components and Eigenvector matrix by principal component analysis of different maize varieties under dry land condition.

\begin{tabular}{lrrrr}
\hline & CI 1 & CI 2 & CI 3 & \multicolumn{1}{c}{ CI 4 } \\
\hline X1 & 0.33 & 0.05 & 0.23 & 0.24 \\
X2 & -0.16 & 0.05 & 0.27 & 0.69 \\
X3 & 0.31 & 0.08 & 0.06 & 0.35 \\
X4 & 0.34 & 0.06 & -0.21 & -0.07 \\
X5 & 0.36 & -0.03 & -0.04 & -0.03 \\
X6 & -0.26 & 0.11 & 0.33 & -0.16 \\
X7 & 0.05 & 0.18 & 0.52 & -0.24 \\
X8 & -0.13 & 0.14 & 0.53 & 0.00 \\
X9 & -0.35 & -0.01 & -0.19 & 0.01 \\
X10 & 0.37 & 0.06 & 0.04 & 0.08 \\
X11 & -0.14 & 0.33 & -0.15 & 0.21 \\
X12 & -0.23 & 0.25 & -0.19 & 0.16 \\
X13 & 0.03 & 0.41 & -0.01 & -0.02 \\
X14 & -0.04 & 0.42 & -0.12 & 0.01 \\
X15 & 0.02 & 0.40 & -0.12 & -0.09 \\
X16 & 0.27 & 0.13 & 0.16 & -0.30 \\
X17 & -0.07 & 0.36 & -0.05 & -0.26 \\
X18 & 0.20 & 0.32 & -0.10 & 0.14 \\
Eigen values & 6.53 & 5.00 & 2.36 & 1.03 \\
Cumulative contribution & 36.26 & 64.01 & 77.13 & 82.88 \\
rate/\% & & & & \\
\hline & & & & \\
\hline
\end{tabular}

X1: Plant height, X2: Stem diameter, X3: SPAD values, X4: Canopy temperature, X5: Stay-green ability, X6: Net photosynthetic rate, X7: Stomatal conductance, X8: Transpiration rate, X9: The ratio of dry weight and fresh weight, X10: Aboveground biomass, X11: Single ear weight, X12: Hundred-grain weight, X13:Ear length, X14: Ear diameter, X15: Axis diameter, X16: Bald length, X17: Ear row number and X18: Row grains.

Based on the above analysis, it can be learned that drought-tolerant varieties do not necessarily saline-alkali tolerant, while saline-alkali tolerant varieties have some degrees of drought tolerance. Both drought and saline-alkali are abiotic stress factors which affect the growth and development of crops are important influencing factors that restrict the yield, quality and 


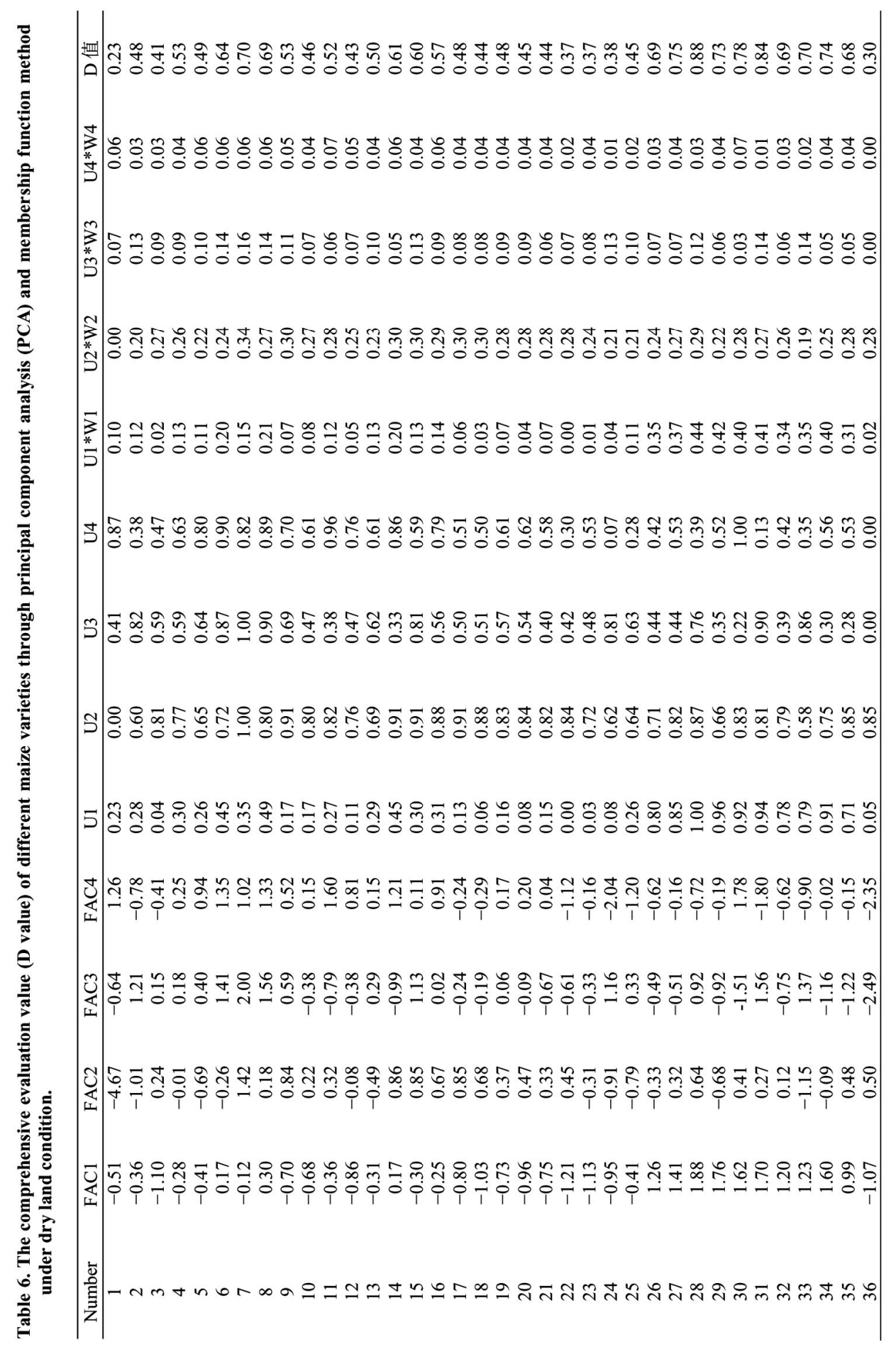




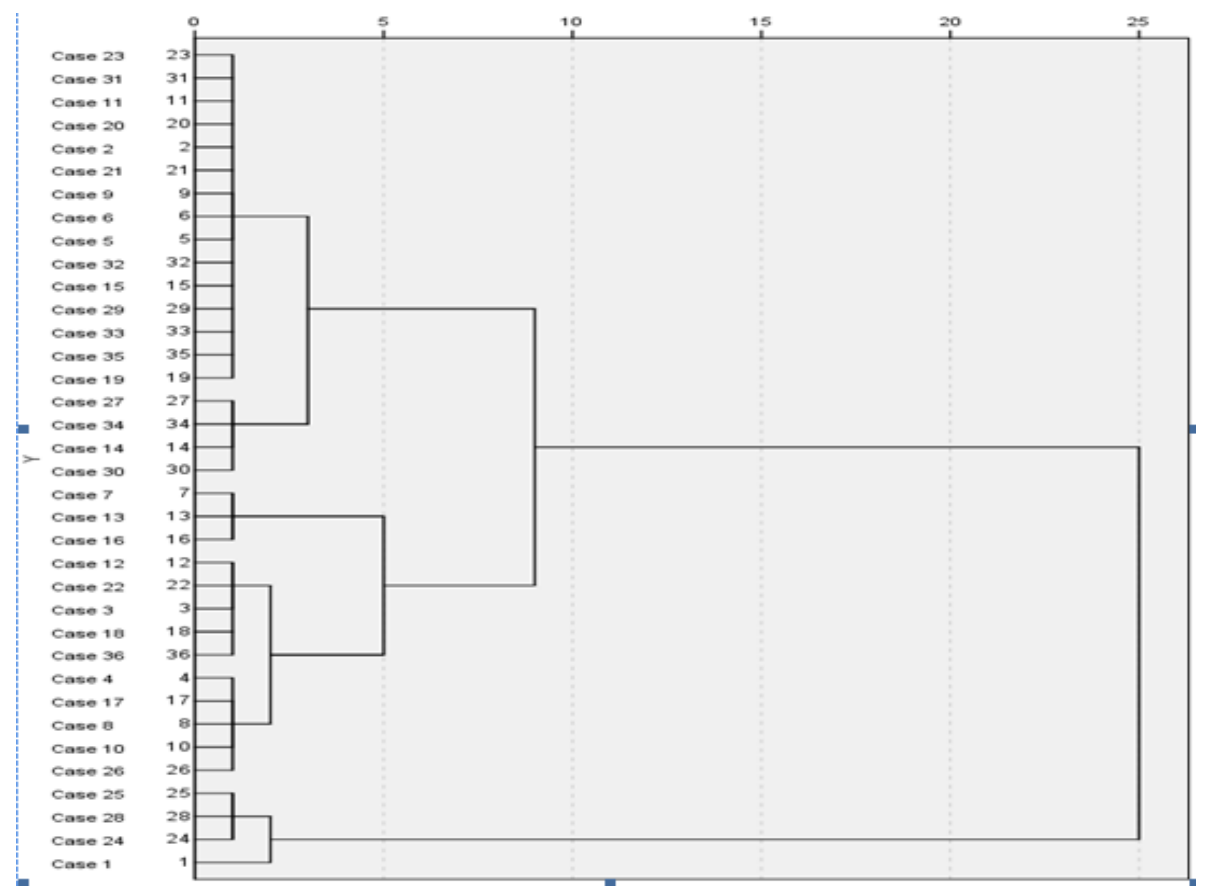

Fig. 3. Cluster analysis of different maize varieties based on D value under saline-alkali condition.

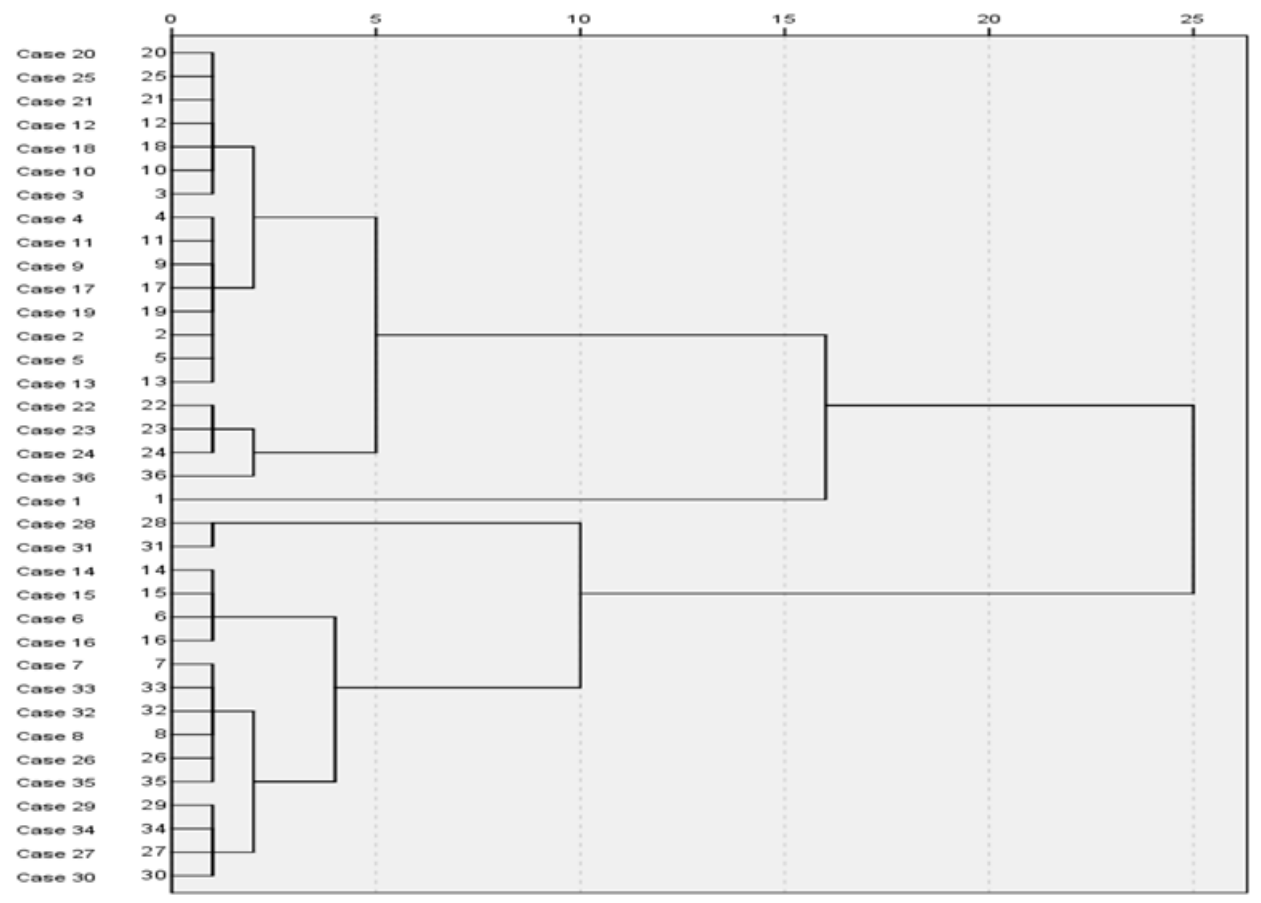

Fig. 4. Cluster analysis of different maize varieties based on D value under dry land condition. 
sustainable development of crops. The study on drought tolerance and saline-alkali tolerance of crops has the significance effects on both agricultural production and ecological environment construction.

In this experiment, the same field management was controlled except for the environmental factors in the experimental field (Ningxia central arid belt and Ningxia Yinbei saline-alkali land, respectively). The results showed that the limited extent of drought-induced crop's growth and development was much larger than the saline-alkali stress. Under drought stress, the average values of agronomic traits, photosynthetic parameters (excluding net photosynthetic rate), stay-green ability, and yield of all maize varieties were significantly lower than those of saline and alkali stress (p < 0.05). Jiang and Huang (2001), Xu et al. (2002a), Abraham et al. (2004), Wang and Huang (2004) and Su et al. (2007) studied the effects of drought and salt stress on plants, the results showed that both drought and saline-alkali stress severely affected the normal growth of plants and differently make the degree of yield reduction, among them: drought stress > saline-alkali stress, which is consistent with the results of this study. This may be due to the fact that the activity and balance of the protective enzyme system in maize were not damaged under saline-alkaline stress conditions, and this balance was destroyed under drought stress, which resulted in the accumulation of reactive oxygen species, initiated and aggravated membrane lipid peroxidation. Studies have shown that when one wants to plant crops with mild salt soils in arid or semi-arid areas, as long as there are certain irrigation conditions to prevent plants from being subjected to drought stress, the plants themselves can maintain their good growth status through "self-regulation".

In this experiment, the stay-green ability of the maize at mature stage under drought stress was significantly lower than that of under saline-alkali stress $(\mathrm{p}<0.05)$, which may be due to the obvious weakening of photosynthesis caused by drought stress and the subsequent water use efficiency decreased, stomatal conductance decreased, and intercellular $\mathrm{CO}_{2}$ concentration decreased. And plants can accumulate a large amount of metal ions such as potassium, sodium, magnesium and calcium ions under salt-alkaline stress conditions, and the magnesium is the only mineral component of chlorophyll molecules. Therefore, the degree of chlorophyll content (chlorosis) induced by drought stress was significantly lower than that of saline-alkali stress. It was also found in this experiment that drought-tolerant lines are not necessarily tolerant to saline-alkali, while strong salt-tolerant lines have some degree of drought tolerance. For example, the lines of A28/1522 and 5178-1/1528 had strong drought tolerance, but when they were planted in saline-alkali soil, the two lines belong to an extremely sensitive group. The lines with strong salt tolerance (including 133-2/1528, H398/1523 and Ning 3-2/1528), all of which have some degree of drought tolerance. This is consistent with the study of spring wheat under water and salt (Xu et al. 2002b).

It may be classified that varieties like 133-2/1528, H398/1523 and Ning3-2/ 1528 were clustered in the high saline-alkali tolerance group, suggesting that they had the highest saline-alkali tolerance capability. The A28/1522 and 5178-1/1528 were clustered in the high drought-tolerant group, suggesting that they had the highest drought tolerance capability. Three saline-alkali tolerance maize lines and two drought tolerance maize lines, which can be used by breeders for the genetic improvement in other maize varieties.

\section{Acknowledgements}

This study was supported by research grants from Fund Program of Financial Support for Special Project of First-Class University in western China for the construction of State Key Laboratory of Ningxia University (GZXM2017001). 


\section{References}

Abraham EM, Huang B, Bonos SA and Meyer WA 2004. Evaluation of drought resistance for texas bluegrass, kentucky bluegrass, and their hybrids. Crop Sci. 44: 746-1753.

Akram M, Ashraf MY, Ahmad R, Waraich EA, Iqbal J and Mohsan M 2010. Screening for salt tolerance in maize (Zea mays L.) hybrids at an early seedling stage. Pak. J. Bot. 42: 141-154.

Bo W, Fu B, Qin G., Xing G. and Wang Y 2017. Evaluation of drought resistance in Iris germanica L. based on subordination function and principal component analysis. Emir. J. Food Agr. 29: 770-778.

Chen DM 2002. Evaluation of salt tolerance of wheat with subordinate function value method. Acta Pedol. Sin. 39: 368-374.

Du Y, Duan ZY, Pan YH, Lei BK, Wan-Li HU, Bin FU, Chen AQ, Chen SH, Yang YX and Jin G.M 2015. Effect of drought stress on growth and activities of antioxidant enzymes of maize seedling. Agric. Res. Arid Areas 33: 124-129.

Fu Y, S. Gao R and Wang ZH 2009. Evaluation of Salt Tolerance of Maize Germplasm in Seedling Stage. J. Maize Sci. 17: 36-39,50.

Glenn EP, Brown JJ, and Blumwald E 1999. Salt tolerance and crop potential of halophytes. Crit. Rev. Plant Sci. 18: 227-255.

Guan GF, Wang YS, Cheng H, Jiang ZY and Fei J 2015. Physiological and biochemical response to drought stress in the leaves of Aegiceras corniculatum and Kandelia obovata. Ecotoxicology 24: 1668-1676.

Hao DR, Cheng YJ, Xu CW, Mao YX, Peng CJ and Xue L 2013. Screening of maize germplasms for salt-tolerance and evaluation of population genetic structure. J. Plant Genet. Resour. 14: 1153-1160 (1158).

Jiang, Y. and Huang B. 2001. Drought and heat stress injury to two cool-season turfgrasses in relation to antioxidant metabolism and lipid peroxidation. Crop Sci. 41: 436-442.

Karamizadeh S, Abdullah SM, Manaf AA, Zamani M and Hooman A 2013. An overview of principal component analysis. J. Signal Inform. Processing 4:173-175.

Kutlu N, Terzi R, Tekeli C, ŞEnel G., Battal P and KadiOğLu A 2009. Changes in anatomical structure and levels of endogenous phytohormones during leaf rolling in Ctenanthe setosa under drought stress. Turk. J. Biol. 33: 115-122.

Lu H and Wang HZ 2004. Governance is probably the use of research progress. Mod. Agric. 8: 10-12.

Rodrí GuezP, Torrecillas A, Morales MA, Ortuño MF and Sánchez-Blanco MJ 2005. Effects of NaCl salinity and water stress on growth and leaf water relations of Asteriscus maritimus plants. Environ. Exp. Bot. 53:113-123.

Shi YH, Wan LQ, Liu JN, Wang YQ, Guo R, Wu XM and Li XL 2010. Analysis of the principal components and the subordinate function of Lolium perenne drought resistance. Acta Agrestia Sin. 18: 669-672.

Su K, Bremer DJ, Keeley SJ and Fry JD 2007. Effects of high temperature and drought on a hybrid bluegrass compared with kentucky bluegrass and tall fescue. Crop Sci. 47: 2152-2161.

Takehisa H, Shimodate T, Fukuta Y, Ueda T, Yano M, Yamaya T, Kameya T and Sato T 2004. Identification of quantitative trait loci for plant growth of rice in paddy field flooded with salt water. Field Crop Res. 89: 85-95.

Trachsel S, Messmer R, Stamp P, Ruta N and Hund A 2010. QTLs for early vigor of tropical maize. Mol. Breeding 25:91-103

Wang ZL and Huang BR 2004. Physiological recovery of Kentucky bluegrass from simultaneous drought and heat stress. Crop Sci. 44: 1729-1736.

Xu X, Zheng G., Deng X, Xu Z and Liu Z 2002a. Comparative study of drought and salt resistance of different genotypical triticinae. Acta Bot Boreali-Occident Sin. 22: 1122-1135.

Xu X, Zheng G., Deng X, Xu Z and Hui H 2002b. Comparison study on osmotic adjustment of accumulation of spring wheat seedlings in water and salt stress. Agri. Res. in the Arid. Areas 20: 52-56. 
Yan F, Gao SR and Yang KJ 2011. Effects of salt stress on several physiological and biochemical indicators in seedling of salt-tolerant line and salt-sensitive line of maize (Zea mays L.). Plant Physiol. J. 47: 459-462.

Yu XJ, Xiao H, Chang-Lin XU, Jing YY and Chai CY 2015. Comparative study on drought tolerance and salt tolerance of medicago ruthenica and medicago varia at seed germination period. J. Plant Genet. Resour. 16: $405-410$.

Zhang HL, Xing X and Zhu L 2017. Comprehensive evaluation on drought-tolerance of maize in mature stage by subordinate function values analysis. J. Maize Sci. 25: 32-39.

Zhang FJ, Zhang KK, Du CZ, Li J, Xing YX, Yang LT and Li YR 2015. Effect of drought stress on anatomical structure and chloroplast ultrastructure in leaves of sugarcane. Sugar Tech. 17: 41-48.

Zhang RH, Xue JQ, Jun PU, Bing Z, Zhang XH and Zheng YJ 2011. Influence of drought stress on plant growth and photosynthetic traits in maize seedlings. Acta Agron. Sin. 37: 521-528.

Manuscript received on 24 August, 2018; revised on 7 June, 2019) 